\section{Gold and Gold Alloy foams}

\section{John Banhart}

Helmholtz Centre Berlin for Materials and Energy

(Hahn-Meitner Institute)*

Glienicker Str. 100, 14109 Berlin, Germany

*Experimental work was carried out at the FraunhoferInstitute for Manufacturing Technology and Applied

Materials Research, Bremen, Germany

\begin{abstract}
The possibilities to manufacture gold-based foams are explored. Gold powder and various powdered alloying elements are mixed with a small volume fraction of a gas-releasing blowing agent. The blend is compacted to a dense precursor, which is then melted in a further step in order to trigger foam formation. We find that gold-silicon alloys containing 2-3 wt.\% of silicon or around 8 wt.\% of germanium can be foamed using $\mathrm{TiH}_{2}$ or $\mathrm{ZrH}_{2}$ as a blowing agent. Foams with about $85 \%$ porosity are obtained.
\end{abstract}

\section{Introduction}

Metals can be foamed applying a variety of processing methods. 'Foaming' means dispersing gas bubbles in a melt and allowing them to arrange into a state of transient equilibrium. A solid metal foam is obtained by solidifying a liquid foam at a given instant, usually near maximum volume expansion [1]. As liquid foams contain gas bubbles completely surrounded by the liquid phase, solid foams are materials with closed porosity. Such materials, especially those based on aluminium alloys, have good mass-specific properties, such as a high elastic stiffness and are therefore being considered useful materials for light-weight construction. Due to their strongly non-linear crush behaviour metallic foams are also good mechanical energy absorbers $[2,3]$. Currently there is a small market for aluminium foams and a few companies producing aluminium alloy foams. Applications range from parts for mobile lifting systems, an 'Ariane V' rocket adaptor and crash absorbers for a light city railway car [4].

Besides foaming, there are a number of other ways to process metals into porous, spongeous or other cellular states: By applying powder metallurgy, investment casting, electroplating or electrodeposition [3,5], just to name the most common ones. As an example, macroporous gold has been produced by filling the interstices of polymer sphere packings with gold powder, pressing the mix, removing the polymer and sintering. The skeletal plates of marine echinoids were used as templates that were then gold-coated chemically to create microporous gold with pore sizes around $15 \mu \mathrm{m}$ [6]. Even nanoporous gold can be manufactured by selective de-alloying of Ag-Au alloys [7] or by using colloidal crystal templates from stacked latex microspheres and filling the interstices with colloidal gold particles [8]. Alternatively, micro-templates can be coated by electrochemical plating to manufacture pure porous gold with up to $77 \%$ porosity [9].

In the following, we will discuss the possibilities to foam gold alloys in the narrow sense described above, i.e. by generating gas bubbles in a liquid gold alloy. Gas bubbles injected into a liquid will float to the surface and burst there unless the melt contains stabilising particles that adhere to the bubble surfaces and prevent the bubbles from merging with each other. Aluminium alloy foams are stable because they contain oxide particles or other non-metallic particles such as silicon carbide [10]. Other metals such as zinc [11], lead [12] or steel [13] can be foamed following the same principles. One particularly successful method comprises mixing aluminium or zinc powder with the gas-releasing blowing agent titanium hydride $\left(\mathrm{TiH}_{2}\right)$, compacting this mixture and melting it thereafter. During melting the blowing agent releases gas and creates bubbles [14]. The oxides present in the melt stem from the oxide films present on and inside the former powder particles and remain in the compacted powder and in the final foam $[10,15,16]$.

When trying to transfer this foaming method to gold alloys, one encounters two problems. First of all, the melting 
point of gold $-1064^{\circ} \mathrm{C}$ - is much higher than the decomposition range of $\mathrm{TiH}_{2}$ - about 350 to $800^{\circ} \mathrm{C}$ [17] and therefore it can be anticipated that $\mathrm{TiH}_{2}$ is not suitable for foaming pure gold. Second, gold does not contain oxides which could stabilise the gas bubbles. Although it is known that the blowing agent particles themselves can act as stabilisers [18], their volume content is usually low and it is expected that additional measures for stabilising gold foams are necessary.

The strategy followed in this work is to alloy gold with a selection of elements in order to both lower the melting point so much that $\mathrm{TiH}_{2}$ can be used as a blowing agent, and to allow the alloying elements to act as stabilisers, either directly or via the generation of non-metallic constituents. In addition to $\mathrm{TiH}_{2}$, two further hydrides were tested, $\mathrm{ZrH}_{2}$ and $\mathrm{MgH}_{2}$.

\section{Experimental}

Gold powder was provided by C. Hafner (Pforzheim, Germany) who used the Plasma Rotating Electrode Process (PREP) to atomise rods of pure gold. The powders were examined by scanning electron microscopy and characterised in a laser particle size analyser. Figure 1 shows two images at different magnifications, illustrating the wide range of particle sizes and the near-spherical shape of most of the particles. This shape is typical for such PREP powders. The particle size distribution in Figure 2 yields a mean value of $\approx 40 \mu \mathrm{m}$ $\left(\mathrm{d}_{50}=34 \mu \mathrm{m}\right)$ and shows that there is a significant fraction of smaller particles residing on the larger ones as

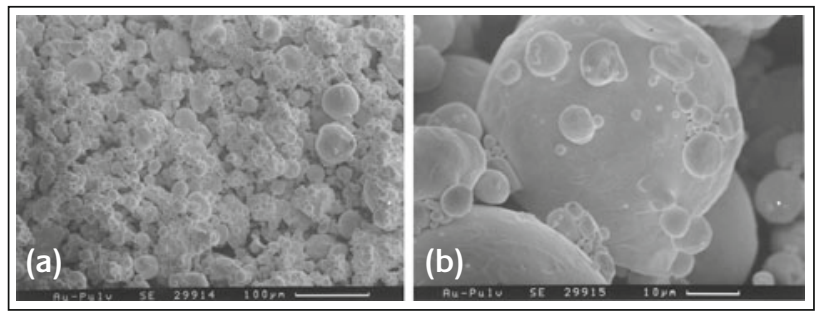

Figure 1

SEM images of gold powder used. (a) low magnification, (b) higher magnification

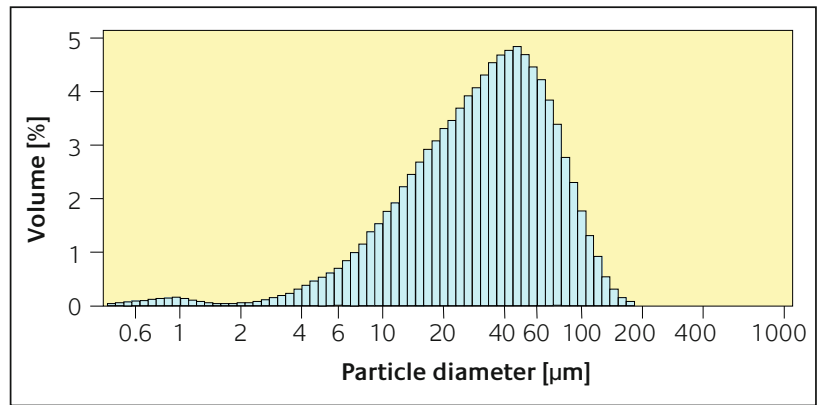

Figure 2

Particle size distribution of the gold powder as obtained by laser particle analysis

satellite particles, see Figure 1b. In addition, some very large particles and flakes were detected. From the experience with aluminium foams it can be expected that these powders should be compactable without encountering major problems.

The alloys were selected by screening the available phase diagrams and searching for systems in which a small addition of an alloying element reduces the melting point considerably

\section{Table 1}

Alloys and blowing agents used for foaming. Extrusion conditions and resulting precursor densities are given. $T_{\text {pre }}=$ temperature of pre-heating; $p_{\text {peak }}$ peak pressure occurring at beginning of pressing; $\rho_{\text {calc }}$ and $\rho_{\text {expt }}$ : calculated and measured experimental density; relative density= $\rho_{\text {exp }} / \rho_{\text {calc }}$; .d.: no determination of density because extruded material was too rough. Asterisk in 1st column: expansion data for these materials given in Figure 4

\begin{tabular}{|c|c|c|c|c|c|c|c|}
\hline no. & $\begin{array}{l}\text { alloy } \\
\text { [wt.\%] }\end{array}$ & $\begin{array}{l}\text { blowing agent } \\
\text { [wt.\%] }\end{array}$ & $\begin{array}{l}\mathrm{T}_{\text {pre }} \\
{\left[{ }^{\circ} \mathrm{C}\right]}\end{array}$ & $\begin{array}{l}\mathrm{p}_{\text {peak }} \\
{[\mathrm{MPa}]}\end{array}$ & $\begin{array}{l}\rho_{\text {calc }} \\
{\left[\mathrm{g} / \mathrm{cm}^{3}\right]}\end{array}$ & $\begin{array}{l}\text { absolute } \\
\rho_{\text {expt. }} \\
{\left[\mathrm{g} / \mathrm{cm}^{3}\right]}\end{array}$ & $\begin{array}{l}\text { relative } \\
\text { [\%] }\end{array}$ \\
\hline 1 & $A u$ & none & 600 & 300 & 19.30 & $19.17 \pm 0.02$ & 99.3 \\
\hline 2 & Au & 1\% TiH & 500 & 525 & 18.63 & $18.37 \pm 0.04$ & 98.6 \\
\hline $5^{*}$ & AuSi1 & $1 \% \mathrm{TiH}$ & 350 & 600 & 17.40 & $17.02 \pm 0.07$ & 97.8 \\
\hline $8^{*}$ & AuSi1.5 & 1\% TiH & 350 & 1125 & 16.85 & $16.40 \pm 0.06$ & 97.3 \\
\hline $13^{*}$ & AuSi1.5 & 1\% TiH & 350 & 675 & 16.85 & $16.50 \pm 0.41$ & 97.9 \\
\hline $14^{*}$ & AuSi2 & 1\% TiH & 350 & 810 & 16.33 & $15.79 \pm 0.06$ & 96.7 \\
\hline 16 & AuSi2 & 1\% TiH & 350 & 1020 & 16.33 & n.d. & n.d. \\
\hline $6^{*}$ & AuSi2 & 1\% TiH & 350 & 1035 & 16.33 & $15.87 \pm 0.05$ & 97.2 \\
\hline $9^{*}$ & AuSi2 & $1 \% \mathrm{ZrH}$ & 300 & 930 & 16.56 & $15.88 \pm 0.1$ & 95.9 \\
\hline $11^{*}$ & AuSi2 & $1 \% \mathrm{MgH}$ & 300 & 1005 & 15.53 & $14.74 \pm 0.06$ & 95 \\
\hline $3^{*}$ & AuSi3 & $1 \% \mathrm{TiH}$ & 450 & 1050 & 15.38 & n.d. & n.d. \\
\hline $12^{*}$ & AuSi2Cu4.5 & $1 \% \mathrm{TiH}$ & 350 & 1050 & 15.61 & $14.62 \pm 0.07$ & 93.7 \\
\hline 4 & AuAl2 & $1 \% \mathrm{TiH}$ & 600 & 187 & 16.65 & $16.84 \pm 0.04$ & 100.0 \\
\hline 7 & AuAl2 & 1\% TiH & 350 & 675 & 16.65 & 16.45 & 98.8 \\
\hline 10 & AuSn5 & $1 \% \mathrm{TiH}$ & 225 & 885 & 17.31 & $17.07 \pm 0.14$ & 98.6 \\
\hline 15 & AuGe8 & $1 \% \mathrm{TiH}$ & 350 & 630 & 15.46 & $14.14 \pm 0.09$ & 91. \\
\hline
\end{tabular}




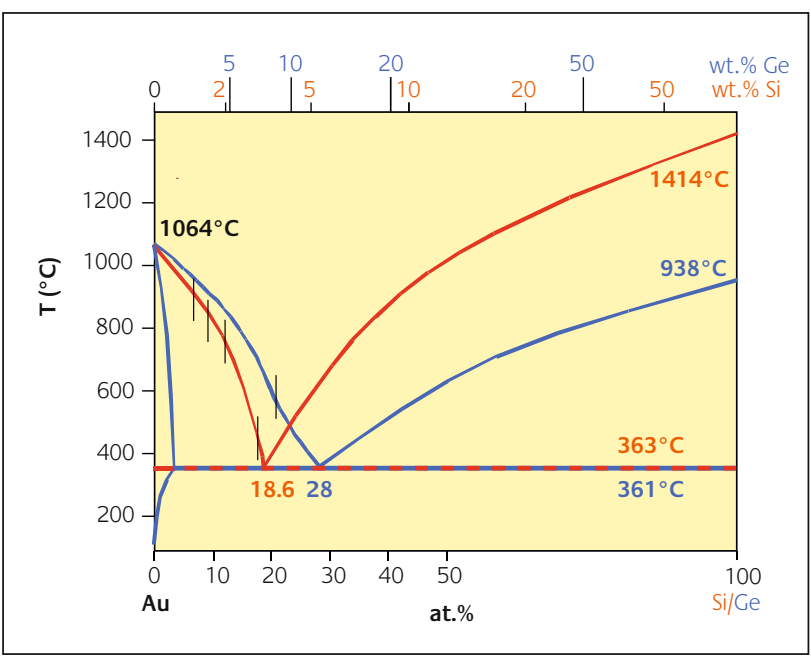

\section{Figure 3}

Phase diagram of Au-Si and Al-Ge alloy systems (redrawn from Ref. 20). Short vertical dashes indicate the compositions used

and yields an extended semi-solid range. Figure 3 shows the phase diagram of the system Au-Si which eventually led to the best results. Small additions in terms of mass lead to the formation of a very deep eutectic that solidifies at $363^{\circ} \mathrm{C}$. At such temperatures and above, the hydrides that are commonly used as blowing agent for aluminium alloys release sufficient amounts of hydrogen. Si is practically insoluble in gold. No stable intermetallic phases are observed, but the existence of metastable phases is known [19].

Other alloy systems selected were Al-Ge, Au-Al, Au-Sn, and the ternary alloy Au-Si-Cu. Al-Ge is very similar to Al-Si with the difference that more $\mathrm{Ge}$ is required to lower the melting point of gold and that $\mathrm{Ge}$ is soluble in $\mathrm{Au}$ at small concentrations, see Figure 3. Both Al and Sn lower the melting temperature of gold, form intermetallic phases with gold and are soluble. The corresponding phase diagrams can be found in Ref. 20.

The blowing agent $\mathrm{TiH}_{2}$ was purchased from Chemetall (Frankfurt, Germany), $\mathrm{ZrH}_{2}$ from Chempur (Karlsruhe, Germany), $\mathrm{MgH}_{2}$ from Coldschmidt (Essen, Germany). All these powders have a size distribution with $d_{50}$ in the range of 13 to $20 \mu \mathrm{m}$.

Powders were mixed in a tumbling mixer for 30 minutes in the fractions given in Table 1. In some cases, mixing was not uniform and some of the lighter powder was found on top of the mixture. In such cases, manual stirring was applied until the powder blend appeared to be visually uniform.

Powder compaction was done by extrusion. For this, $100 \mathrm{~g}$ of powder mixture were first pre-compacted at room temperature and $250 \mathrm{kN}$ pressure to tablets of $29 \mathrm{~mm}$ diameter in a cylindrical die. Two (three for experiment no. 16) such tablets were loaded into the extrusion die followed by a graphite disk of the same size. The die was pre-heated for about 2 hours at the temperature $T_{\text {pre }}$ given in Table 1, after which the material was extruded to wires of $9.5 \mathrm{~mm}$ diameter. This corresponds to an extrusion ratio of 9.3. The extrusion peak pressure $p_{\text {peak }}$ ranged between about 200 and 1100 MPa. After extrusion, the density was determined by buoyancy measurement, see Table 1.

For foaming, a furnace was pre-heated to a temperature $50 \mathrm{~K}$ above the liquidus temperature of the respective alloy and was left there for some time for temperature equilibration. After this, a piece of the pressed material, about $1 \mathrm{~cm}$ long, was put into the furnace and was left there for heating and foaming. The sample was exposed to air all the time. The foaming process was followed by visual observation. In some cases the pressed material started to foam. The foaming process appeared similar to the foaming of other metals: after an initial expansion stage, a maximum volume was reached. Then, foam collapse was observed which eventually destroyed the foam. After a picture of the overall foaming course had been obtained, the foaming process was interrupted in further experiments at or near maximum expansion by taking the samples out of the furnace and cooling them in a stream of cool air. Foams which could be preserved in a state near maximum expansion were used for further analysis. After measuring the density (or porosity), the foam was sectioned and the cell structure observed.

\section{Results}

Extrusion led to a good consolidation of the powder to wires in most cases. The peak pressure needed to initiate flow shows considerable scatter. Lower pre-heating temperatures required higher pressures in most cases. Except for experiments 3 and 16 the surface of the wires was smooth and the wires all showed some bending ductility. The density values given in Table 1 indicate that densification was best in pure gold, followed by Au-Al and Au-Sn. The powder mixtures alloyed with Si and Ge showed the highest level of porosity.

In the foaming experiments the different alloys behaved differently. No notable or just a small volume expansion was observed for pure Au, Au-Al and Au-Sn alloys. After cutting, these specimens showed some pores of different sizes distributed all over the sample, but the total pore volume was small and pore distribution was irregular. It was obvious that these alloys in the current composition are unsuitable for foaming.

In contrast, Al-Si and Al-Ge yielded much better results. In all cases, the extruded powder mixtures started to expand after some time in the furnace. Foaming took place in a smooth and continuous way as it is known from aluminium alloys. The resulting foams at near-maximum expansion had expanded to 2 to 6 times the original precursor volume. The volume expansion factor defined as the ratio of foam volume to original precursor volume depended on the silicon content in the sample, see Figure 4 . Below a silicon content of $2 \%$ expansion factors up to 3.5 are reached, whereas for $2 \%$ Si an expansion factor of more than 6 was found. For more than $2 \%$ Si the expansion factor appears to remain at this level. Replacing $\mathrm{TiH}_{2}$ by $\mathrm{ZrH}_{2}$ did not change the foaming behaviour and produced very similar foams, whereas $\mathrm{MgH}_{2}$ did not yield satisfactory results at all. Addition of $4.5 \%$ Cu did not have a 


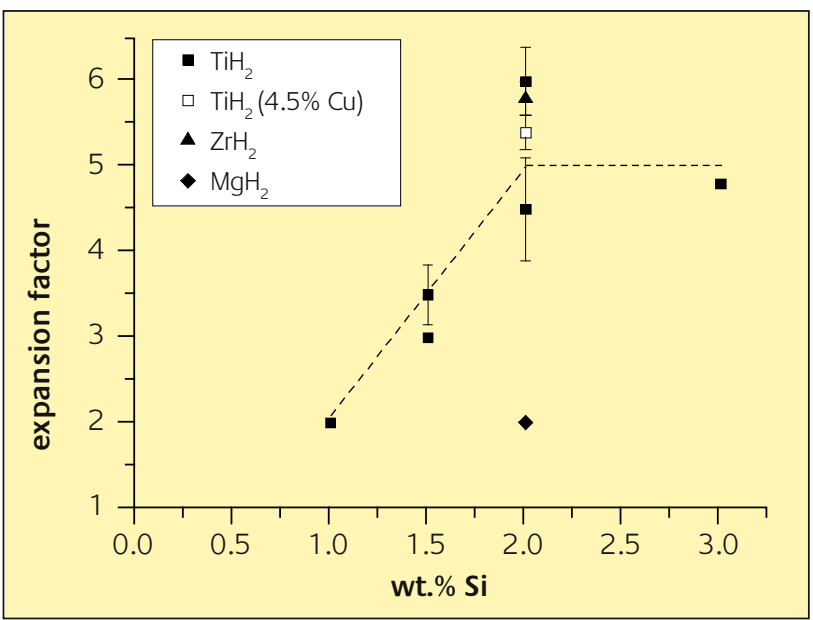

\section{Figure 4}

Volume expansion factors for various Au-Si foams and a AuSizCu4.5 alloy foam produced with different blowing agents. Each point represents foaming experiments based on one of the extruded alloys marked by an asterisk in Table 1. Error bars specify a range whenever more than one experiment was carried out on the same batch of extruded material. The dotted line is just a guide line

pronounced effect on foaming. The sample containing 8 wt.\% Ge expanded to 3.5 times the original volume.

The best foam obtained is shown in Figure 5a. This structure which has expanded to about 6 times the original volume - and therefore contains about $84 \%$ porosity or, equivalently, has a mean density around $2.5 \mathrm{~g} / \mathrm{cm}^{3}$ - shows a diversity of pore sizes. The largest visible pore is about $4 \mathrm{~mm}$ in diameter.

The foam did not appear shiny since both the outer surface and the interior cell walls were rough. Immediately after foaming and cooling the foams had a silvery colour. After a few hours at room temperature the colour changed to what can be described best as light ochre. The micrograph in Figure 6 reveals that the cell walls are rather jagged. The high volume fraction of silicon ( 2 wt.\% corresponding to about 14 vol.\%) is reflected by the dark silicon phase in the light gold matrix.

Au-Ge foams assumed a clear bluish hue after foaming which could be polished off. The copper containing AuSi2Cu4.5 alloy had turned into a silvery colour.

\section{Discussion}

The study showed that Au-Si alloys can be foamed by using a procedure very similar to that used to foam aluminium or zinc alloys. Both $\mathrm{TiH}_{2}$ and $\mathrm{ZrH}_{2}$ are well adapted to the low melting point of the deep eutectic of this alloy. Addition of silicon leads to a pronounced stability of the foam. It could not be clarified how silicon acts as stabiliser. As the silicon powder contains 0.6 wt.\% of oxygen - as measured by hot gas extraction - one could assume that silicon oxide acts in a way similar as aluminium oxide in aluminium foams. The same applies to the Au-Ge alloy since Au-Si and Au-Ge have very similar phase diagrams and the germanium powder most likely also contained oxygen picked up during powder manufacture.

Applying standards of state-of-the-art aluminium foams, the pore structure is inhomogeneous and the quality of the foam is not optimal as one sees when one compares Figure $5 a$ with Figure 5b that shows an aluminium-based foam. However, it is an indication of good foaming behaviour that the entire cross section of the foam appears to have foamed in the same way and that there is no pronounced zone of drainage at the bottom of the foam, although the density of the metal is so high. From the foaming behaviour of other metals it is known that inferior foaming caused by insufficient compaction of the powders or an unsuitable choice of powders often leads to irregular pores and heavy drainage, see e.g. Ref. 10. This is obviously not the case here.

The presence of silicon together with the roughness of the surface can be held responsible for the colour of the Au-Si foam. The colour change of Au-Si during room temperature storage has also been confirmed for non-porous ingots prepared under argon [21]. It was found that only the surface changes its colour, whereas deeper lying layers remain silvery and that the effect is reversible when the alloy is heated again. Most likely, the solidified alloy contains non-equilibrium
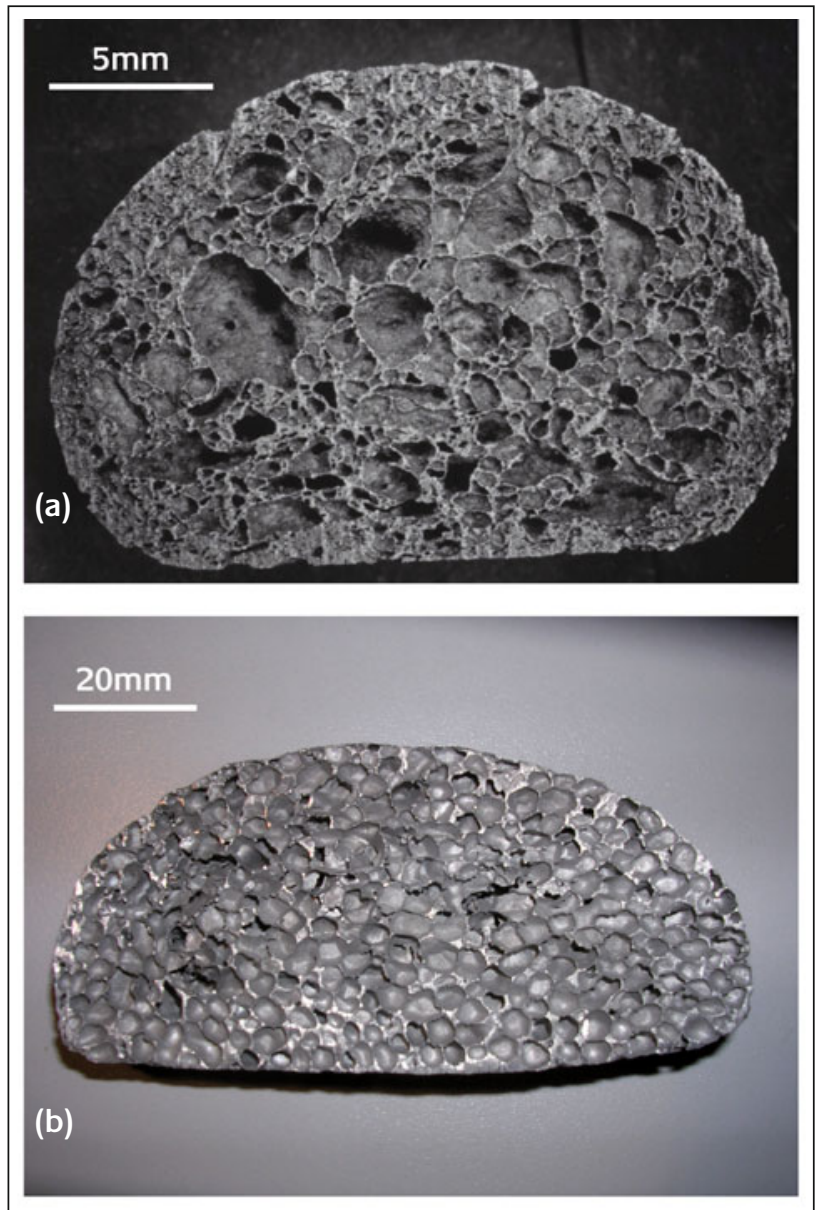

\section{Figure 5}

(a) AuSi2 foam expanded to 6 times the volume of the extruded precursor. Maximum width of foam is $22.5 \mathrm{~mm}$. Precursor no. 6 in Table 1 was used. (b) AuSi6Cu4 foam expanded to 8 times the volume of the extruded precursor 


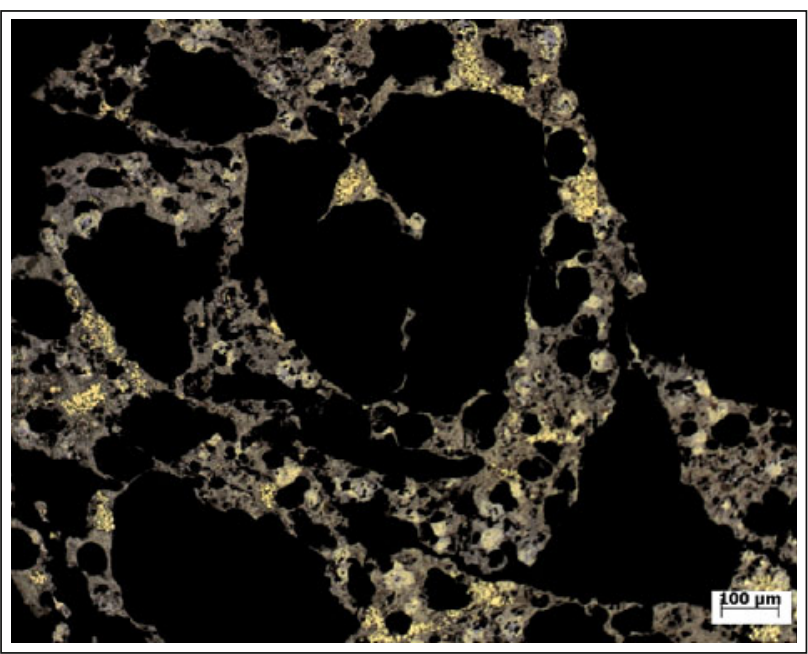

\section{Figure 6}

Microstructure of AuSiz foam shown in Figure 5. Sample ion-polished and imaged by light microscopy

intermetallic phases that have a silvery colour and diffusioncontrolled processes cause the decomposition of these phases into the gold/silicon eutectic at the surface. Similar colour changes have been described for rapidly solidified AuGe-Si-based solders, however, not at room temperature but at $285^{\circ} \mathrm{C}$ [22].

The colours of all the alloys investigated in the current study deviated from those of the usual commercial alloys, see Ref. 23, since our alloys have been selected to optimise foaming behaviour. Moreover, the surfaces are rough and partially oxidised. As not only the occurrence of intermetallics but also that of oxides or other patinas can produce a wide range of colours [24], there are many possible reasons for the observed colours in the foams investigated. Even the blowing agents could lead to further colour shifts, since after decomposition the elements $\mathrm{Ti}$ or $\mathrm{Zr}$ remain in the metallic matrix.

For two reasons it can be assumed that the quality of the gold alloy foams could be improved by further optimising the process parameters. First, the parameters applied in this study merely represent a first guess based on some theoretical considerations and the experience with other metals. Second, experience also shows that the powder selection and compaction process contains many hidden parameters which have to fine-tuned. The blowing agent used in this study was untreated hydride powder. Pre-treatment of these powders could lead to a better coordination of gas release and alloy melting, which in turn could further improve the foaming behaviour as it is known for aluminium alloys, see e.g. Ref. 25.

\section{Conclusions}

Low-melting Au-Si and Au-Ge alloys can be foamed to about 6 times the volume of the starting material using $\mathrm{TiH}_{2}$ or $\mathrm{ZrH}_{2}$ as a blowing agent. Pore sizes are comparable to those of aluminium foams, while the less uniform pore structure reflects a more provisional status of development. It was shown that foaming a pure metal such as gold without stabilising particles is not possible and therefore alloying other metals to gold is a necessary pre-condition.

It came as a disappointment that it was not possible to foam gold without strongly interfering with its visual appearance. All additives changed the colour of gold more than was hoped initially. It was not possible to reduce the amount of additives to below a level that would affect the appearance of the metal in such a marginal way that it could be used for jewellery or design objects as originally planned. However, there might be some use for such foams for other industrial sectors: Electrical connectors with unique functionalities, medical implants or materials helping to heat plasmas by laser radiation are just some examples that have been suggested in discussions.

\section{Acknowledgement}

Dr. G. Wagner of C. Hafner in Pforzheim, Germany generously funded a part of this work, provided materials and helped with fruitful discussions.

\section{About the author}

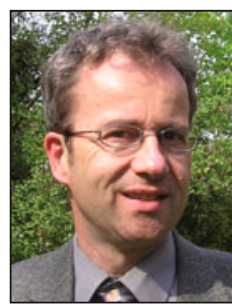

John Banhart is a professor in the Faculty of Materials Science and Technology at the Technical University of Berlin and head of the Department of Engineering Materials of the Helmholtz Centre for Materials and Energy (formerly HahnMeitner-Institute) in Berlin. Current research interests are aluminium alloys, metal foams and $X$ ray and neutron imaging methods. He is a physicist and earned his PhD in physical chemistry at the University of Munich in 1989. He worked at the Fraunhofer-Institute in Bremen for 10 years where a process for foaming metals was developed in close cooperation with industry.

Email: banhart@helmholtz-berlin.de

\section{References}

1 J. Banhart and D. Weaire, On the road again - metal foams find favour, Physics Today, 2003, 55, 37-42

2 M.F. Ashby, A.G. Evans, N.A. Fleck, L.J. Gibson, J.W. Hutchinson and H.N.G. Wadley, Metal foams - A design guide, Butterworth-Heinemann, Boston (2000)

3 J. Banhart, Manufacture, characterisation and application of cellular metals and metallic foams, Progress in Materials Science, 2001, 46, 559-632

4 L.P. Lefebvre, J. Banhart, D. Dunand, Porous metals and metallic foams current status and developments, Advanced Engineering Materials, 2008, $10,775-787$ 
5 H.P. Degischer and B. Kriszt, Handbook of cellular metals, Wiley-VCH, Weinheim (2002)

6 F.C. Meldrum and R. Seshadri, Porous gold structures through templating by echinoid skeletal plates, Chemical Communications, 2000, 29-30

7 J. Erlebacher, M.J. Aziz, A. Karma, N. Dimitrov, and K. Sieradzki, Evolution of nanoporosity in dealloying, Nature, 2001, 410, 450-453

8 O.D. Velev, P.M. Tessier, A.M. Lenhoff, E.W. Kaler, A class of porous metallic nanostructures, Nature, 1999, 401, 548-548

9 K. Nagai, D. Wada, M. Nakai, T. Norimatsu, Electrochemical fabrication of low-density metal foam with mono-dispersed-sized micro and submicrometre pore, Fusion Science and Technology, 2006, 49, 686-690

10 J. Banhart, Metal foams: production and stability, Advanced Engineering Materials, 2006, 8, 781-794

11 J. Banhart, D. Bellmann, and H. Clemens, Investigation of metal foam formation by microscopy and ultra small-angle neutron scattering, Acta Materialia, 2001, 49, 3409-3420

12 A. Irretier and J. Banhart, Lead and lead alloy foams, Acta Materialia, 2005, 53, 4903-4917

13 H.D. Kunze and M. Knüwer, About the foamability of iron - carbon alloys, Steel Research, 1999, 70, 513-518

14 C.J. Yu, H. Eifert, J. Banhart, and J. Baumeister, Metal foaming by a powder metallurgical method: production, properties and applications, Journal of Materials Research and Innovations 1998, 2, 181-188
15 C. Körner, M. Arnold, and R.F. Singer, Metal foam stabilization by oxide network particles, Materials Science and Engineering A, 2005, 396, 28-40

16 A. Dudka, F. García-Moreno, N. Wanderka, J. Banhart, Structure and distribution of oxides in aluminium foam, Acta Materialia, 2008, 56, 3990-4001

17 B. Matijasevic, J. Banhart, S. Fiechter, O. Görke, and N. Wanderka, Modification of titanium hydride for improved aluminium foam manufacture, Acta Materialia, 2006, 54, 1887-1900

18 V. Gergely, D.C. Curran, and T.W. Clyne, The FOAMCARP process: foaming of aluminium MMCs by the chalk-aluminium reaction in precursors, Composites Science and Technology, 2003, 63, 2301-2310

19 B. Predel, Au-Si (gold-silicon), Landolt-Börnstein - New Series, Group IV Physical Chemistry, Vol. 5a, Springer-Verlag (1991)

20 T.B. Massalski, Binary Phase Diagrams, ASM Information Society, Materials Park (1986)

21 S. Mechler, Private communication (2008)

22 D.M. Jacobsen, S.P.S. Sangha, A low melting poind solder for 22 carat yellow gold, Gold Bulletin, 1996, 29, 3-9

23 C. Cretu and E. van der Lingen, Coloured gold alloys, Gold Bulletin, 1999, $32,31-40$

24 C.W. Corti, Blue, black and purple! The special colours of gold, 5th International Jewellery Symposium, St. Petersburg, 3. July 2006

25 B. Matijasevic and J. Banhart, Improvement of aluminium foam technology by tailoring of blowing agent, Scripta Materialia, 2006, 54, 503-508 